\title{
Corrosión de tuberías en instalaciones de edificación
}

\author{
Prof. Dr. JOSE CALLEJA - IETCC - MADRID \\ LIC. ENRIQUE FERNANDEZ MOLINA - Scdad. TUDELA-VEGUIN - ASTURIAS
}

\section{$R E S U M E N$}

Se estudia en este trabajo el comportamiento a un año, aproximadamente, de tubo negro $y$ tubo galvanizado empleados para conducciones de agua en instalaciones de edificación, tanto desnudos como previamente envueltos en papel de periódico -según prácticas locales-, aesde el punto de vista de su corrosion en contacto con morteros $y$ hormigones de cemento, en los que normalmente se encuentran embebidos.

Los resultadios parciales y provisionales dei estudio hasta el momento presente, así como su interpretación $y$ valoración, $y$ las conclusiones emanadas de los mismos, ponen de relieve lo inadecuado $y$ perjudicial de tales prácticas $y$ aconsejan renunciar a las mismas.

En lo que se refiere a la influenciai de los cementos PA-350 con adición de escorias siderúrgicas $y$ S-II-350 del Pliego RC-75, utilizados en el estudio, los resultados no muestran diferencia de comportamiento entre unos $y$ otros, en las condiciones experimentales tenidas en cuenta y al tiempo de observación de un año, en el sentido de que ninguno de ambos cementos causa corrosion en los tubos negros o galvanizados desnudos.

Los resultados ponen también de manifiesto la importancia de la adecuación de los materiales $y$ del cumplimiento de las reglas de buena práctica en la construcción, sí se quiere evitar el mal comportamiento de unos $u$ otros de dichos tubos en instalaciones de conducción de agua en edificación, desde el punto de vista de su. durabilidad frente a la corrosión.

\author{
$S U M M A R Y$
}

This paper deals with the behaviour during about one year, of cast-iron and galvanized cast-iron tubes used for water pipes in building instalations, either as such or previously wrapped up in newspaper -according to some local practices-. from the viewpoint of their corrosion in contact with cement mortars and concretes in which they are normally embedded.

Provisional practical results of the study up to date, as well as their interpretation and evaluation and conclusions drawn from them emphasize how inadequate and damaging such practices may be and lead to reject their application.

As far as the influence of PA-350 cements containing blast-furnace slags, and $S-I I-350$ cements (blas-furnace slag cements) are concerned, which have been used in the work and are specified in Spanish Cement Standaras RC-75, the results do not show any difference in behaviour between both cements, in the sense that neither the one nor the other cause corrosion of not wrapped. cast-iron tubes, either galvanized or not, in the experimental working conditions, when observed after one year of embedment.

The results also show the importance of using adequate materials and accomplishing regulations of good practice in building, to avoid the bad. behaviour of either of the mentioned tubes in water pipes, as far as their durability against corrosion is concerned.

\section{INTRODUCCION}

Es de sobra conocido, por padecido, el problema de las averías en las conducciones de agua de las instalaciones de edificación en servicio -acometidas y desagües, es decir, fontanería y saneamiento, así como calefacción-, consistentes en escapes debidos a roturas por perforación, con los consiguientes trastornos de todo tipo.

Estas perforaciones, bastante localizadas, responden a procesos de corrosión externa de tramos de tubería, de longitud variable, aunque generalmene cortos - rara vez de más de 1 metro-.

La corrosión se produce tanto en tramos horizontales como verticales de tuberías enterradas, embebidas o empotradas, si bien parece tener lugar con mayor frecuencia en los tramos horizontales. 
En éstos se suelen instalar las tuberías, en el mejor de los casos, embebidas en una matriz de mortero o de hormigón de forjados o pisos sobre el cual se coloca después el solado o pavimento. En estos casos se suele atribuir la corrosión de los tubos de acero negro o galvanizado, desnudos o recubiertos de algún modo, a la acción de determinados constituyentes de los cementos y, por ende, a los propios cementos que los contienen.

En este sentido han sido y son objeto de sospecha los cementos PA (sin distinción) del Pliego RC-75 (1) en todas sus categorías resistentes, por una parte, ya que son los que por definición pueden contener hasta un 20 por ciento de adiciones activas y, por otra parte, por ser los más utilizados en construcción y edificación, tanto con fines estructurales como de albañilería.

Dentro de las adiciones activas que pueden contener los cementos PA, las escorias siderúrgicas -y en menor medida las cenizas volantes-, son las más sospechosas de poder inicar o favorecer fenómenos de corrosión en las tuberías embebidas en morteros hechos con dichos cementos.

No obstante, hay que señalar que tales fenómenos de corrosión también se observan, y sus consecuencias también se padecen, en el caso de tuberías simplemente enterradas en algunos suelos de plantas bajas y sótanos, y sin contacto con mortero de cemento. En este caso la corrosión puede -y suele- afectar a tramos más largos de tubería.

En cuanto a los efectos y consecuencias de las averías por corrosión, la importancia es distinta para los afectados - constructor o propietario-, según las circunstancias: menor en el caso de tuberías enterradas en suelos sin mortero de cemento, y mayor en el caso de tubos embebidos en forjados de piso o planta, y embebidos o empotrados en paramentos verticales de cerramiento o partición.

Pero, en general, y como suele suceder en otras situaciones, la responsabilidad de las averías por corrosión se suele atribuir, como queda señalado, al cemento, antes que a los elementos corroídos, o a otros materiales, o a la propia ejecución de la obra. La realidad, no obstante, es que, además de en el cemento -y con frecuencia más y antes que en éste-, la causa de la corrosión reside en una mala o inadecuada ejecución de las obras. Y, como casi siempre y en circunstancias similares, las averías de fugas por perforación debida a corrosión de tuberías, obedecen a una conjunción de concausas, siendo infrecuentes los casos en que con toda claridad puedan atribuirse con sobrada razón a una sola de ellas.

\section{ANTECEDENTES}

Conocido el hecho de las averías en las conducciones de agua por perforaciones debidas a fenómenos de corrosión, algunos prácticos de la construcción, en determinadas zonas del país, tratan de evitarlos por procedimientos empíricos, más intuitivos que racionales y no siempre eficaces; antes bien, equivocados y perjudiciales en muchos casos.

Es relativamente frecuente el intentar evitar la corrosión de las tuberías protegiéndolas con recubrimientos que a priori se juzgan protectores, pero que a posteriori resultan serlo o no.

Es evidente que para casi todo tipo de protección metálica $-\mathrm{y}$ sobre todo de tuberíasen las más diversas circunstancias, existen hoy día procedimientos de recubrimiento que, independientemente de su precio, son fáciles de aplicar y, por lo tanto, de aplicación sugestiva; y hasta eficaces, si son adecuados y se aplican bien. 
Pero sucede que, a causa de la "extrapolación" y por motivos de baratura -y, por supuesto, de desconocimiento-, a veces se aplican "fácilmente" recubrimientos inadecuados y, además, mal. Tal es el caso, como se indicaba, de algunos prácticos de la construcción, de ciertas regiones del país, los cuales acostumbran a "envolver" las tuberías en papel que, en general, procede de periódicos viejos.

Indudablemente esto supone una variable inopinada en cuanto a las posibilidades de corrosión o protección de las tuberías de acero negro o galvanizado utilizadas en las instalaciones de edificación y embebidas, mejor o peor, en morteros u hormigones de diferentes tipos y clases de cemento.

\section{JUSTIFICACION DEL TRABAJO}

De acuerdo con lo expuesto en la Introducción y en los Antecedentes que preceden, tres son las variajbles principales que justifican el presente trabajo de observación experimental con fines prácticos inmediatos: i) la naturaleza de los cementos contenidos en los morteros y hormigones que embeben a las tuberías; ii) la naturaleza de las propias tuberías -de su superficie-; y iii)) la eficacia — ineficacia- de recubrir éstas con papeles de periódico, antes de embeberlas en los conglomerados.

Una observación previa importante es que la experimentación -de laboratorio- ha sido llevada a cabo en condiciones que pudieran calificarse de "ideales", en el sentido de que el material que embebe a las tuberías - hormigón - ha sido de buena calidad en cuanto a diseño, materiales y ejecución -véanse las fotografías-; que el recubrimiento de las tuberías con papel de periódico -en su caso- se ha efectuado con todo euidado; y que la confección, el curado y la conservación de las probetas se han llevado a cabo bajo el más estricto control.

Esto quiere decir que de los resultados obtenidos tienen más valor los negativos; esto es, que a un comportamiento dado de las tuberías en las condiciones experimentales de laboratorio que son las más suaves - véase la parte Experimental-, debe corresponder, en buena lógica, un comportamiento peor en las condiciones reales de las obras. Lo cual equivale a decir que los resultados de la experimentación dejan las conclusiones del lado de la inseguridad, en el sentido de que, si una tubería se comporta mal en las condiciones experimentales de laboratorio, tiene grandes probabilidades de comportarse aún peor en las condiciones reales de obra; mientras que si se comporta bien en las primeras, no hay garantía de que lo haga igual en las segundas, dado lo incontrolable y hasta lo desconocido de las mismas, en general.

No obstante, puede haber conclusiones claras en cuanto a la práctica del envolvimiento de las tuberías con papel de periódico en las obras, y al efecto que tal práctica ejerce sobre las tuberías negras y galvanizadas.

Asimismo se ha considerado probable, pero no seguro, lograr alguna conclusión respecto de la influencia del tipo de cemento, en las condiciones experimentales establecidas, sobre la corrosión de las tuberías.

Con tales miras se llevó a cabo la investigación, se repite que de tipo meramente pragmático y de resultados inmedatos, tal y como el planteamiento del problema y la urgencia de su solución exigen.

\section{PARTE EXPERIMENTAL}

\subsection{Probetas}

Consistieron en prismas de hormigón de $12 \times 12 \times 60 \mathrm{~cm}$, en cuyo eje se encastraron al 
enmoldar tubos de $1 / 2$ pulgada, de longitud algo mayor que la de la probeta, de modo que sobresalieran unos $5 \mathrm{~cm}$ por uno de los extremos de ésta, quedando por el interior a una distancia análoga del otro extremo de la misma.

\subsection{Hormigón}

En todos los casos fue de la composición y de las características siguientes:

\begin{tabular}{lrc} 
Cemento & 350 & $\mathrm{~kg} / \mathrm{m}^{3}$ \\
Arido silícico 6/12 & 767 & $"$ \\
Arena silícica & 1.150 & $"$ \\
Agua & 200 litros \\
Relación agua/cemento & \multicolumn{2}{c}{0,57} \\
Asiento en cono Abrams & $8 \mathrm{~cm}$
\end{tabular}

\subsection{Aridos}

Respondieron en todo caso a las siguientes granulometrías y características:

\begin{tabular}{|c|cc|}
\hline Luz del tamiz $(\mathrm{mm})$ & Arido $6 / 12$ & Arena \\
& & que pasa \\
\hline 0,149 & 0,0 & 5,5 \\
0,297 & 0,0 & 68,0 \\
0,595 & 0,0 & 81,6 \\
1,19 & 0,0 & 91,0 \\
2,38 & 0,0 & 98,5 \\
4,76 & 1,0 & 100,0 \\
6,35 & 39,4 & - \\
8,0 & 74,3 & - \\
9,5 & 98,0 & - \\
12,7 & 99,8 & 2,30 \\
Módulo de finura & 6,01 & - \\
Coeficiente de forma & 0,18 & 2,64 \\
Peso específico & 2,63 & Silícica \\
Naturaleza & Canto rodado \\
& silícico & \\
\hline
\end{tabular}

\subsection{Cementos}

Se utilizaron tres tipos de cemento, de acuerdo con el Pliego RC-75 (1): un cemento portland P-450, un cemento portland con adiciones activas PA-350 conteniendo escoria siderúrgica, $\mathrm{y}$ un cemento siderúrgico S-II-350.

Las razones para utilizar estos cementos fueron las siguientes: el P-450 se empleó por tratarse de un cemento sin adiciones $-\mathrm{y}$ por lo tanto sin aportación de eventuales elementos corrosivos por parte de éstas-, así como por ser de gran resistencia mecánica y suministrar una abundante reserva alcalina a la pasta cementante. En una palabra, por ser un cemento potencialmente buen protector del hierro y del acero.

El cemento PA-350 con escoria siderúrgica se utilizó por ser, como se indicaba en la In- 
troducción, de gran consumo en construcción y en edificación, y porque la escoria podría influir en la corrosión de los tubos. Y el S-II-350 se empleó por ser un cemento siderúrgico con contenido medio de escoria entre el S-I-350 y el S-III-350 (y superior al del PA-350 utilizado), y tener resistencia mecánica suficiente como para poder ser usado en construcción.

\subsection{Tubos}

Se utilizó tubo negro y tubo galvanizado de $1 / 2$ pulgada tanto desnudo como envuelto en papel de periódico, en cada caso, como es costumbre según algunas prácticas locales.

\subsection{Curado y conservación}

Una parte de las probetas se almacenó, una vez sacadas de los moldes a las 24 horas de su amasado y enmoldado, en una cámara húmeda a temperatura de $20 \pm 2^{\circ} \mathrm{C}$ y humedad de $95-100 \%$ HR.

Otra parte se conservó en una dependencia del laboratorio, en las condiciones ambientales del mismo, ni medidas ni controladas.

\subsection{Prismas de ensayo}

De acuerdo con los materiales y condiciones descritos, del 4 al 30-X-80 se fabricaron los siguientes prismas con tubos engastados, los cuales se conservaron hasta las fechas para su observación (habiendo sido la primera el 27-XI-81, al cabo de 1 año y 1 mes, aproximadamente), en las condiciones que se indican.

\begin{tabular}{|c|c|c|c|c|c|c|}
\hline \multicolumn{2}{|c|}{ PRISMAS DE ENSAYO } & \multicolumn{2}{|c|}{ CURADOS EN } & \multirow{2}{*}{$\begin{array}{l}\text { CEMENTOS } \\
\text { Tipo-Categoría }\end{array}$} & \multicolumn{2}{|c|}{ TUBOS } \\
\hline Designados & Fábricados & Cámara & Ambiente & & Material & Estado \\
\hline 1 & 3 & 2 & 1 & PA-350 (esc.) & negro & desnudo \\
\hline 2 & 3 & 2 & 1 & $"$ & galvan. & $"$ \\
\hline 3 & 3 & 2 & 1 & $"$ & $"$ & envuelto \\
\hline 4 & 3 & 2 & 1 & $"$ & negro & $"$ \\
\hline S 1 & 3 & 2 & 1 & S-II-350 & galvan. & desnudo \\
\hline S 2 & 3 & 2 & 1 & $"$ & $"$ & envuelto \\
\hline S 3 & 3 & 2 & 1 & $"$ & negro & desnudo \\
\hline S 4 & 3 & 2 & 1 & $"$ & $"$ & envuelto \\
\hline$?$ & 3 & 2 & 1 & $P-450$ & negro & envuelto \\
\hline$?$ & 3 & 2 & 1 & $"$ & $"$ & desnudo \\
\hline$?$ & 3 & 2 & 1 & $"$ & galvan. & envuelto \\
\hline$?$ & 3 & 2 & 1 & $"$ & $"$ & desnudo \\
\hline
\end{tabular}

Cada prisma se marcó con su designación, y además con el tipo de cemento, clase y condición del tubo, forma de colocación en el curado (vertical u horizontal), así como en la conservación, y con la fecha de fabricación (fotografías 1,2 y 3). 


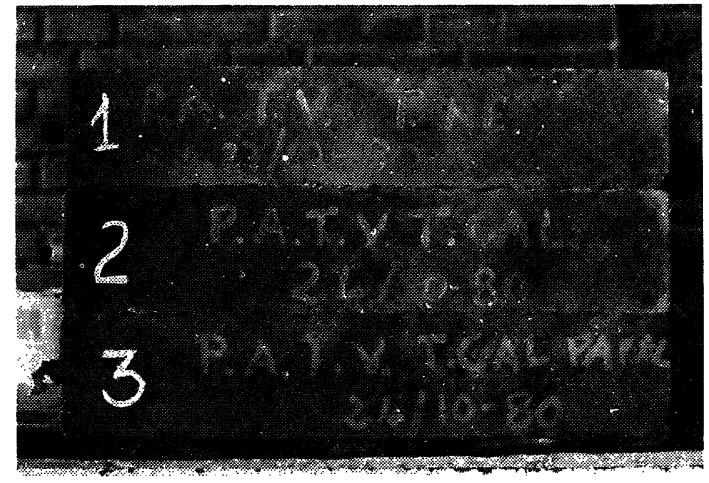

Fotografía 1

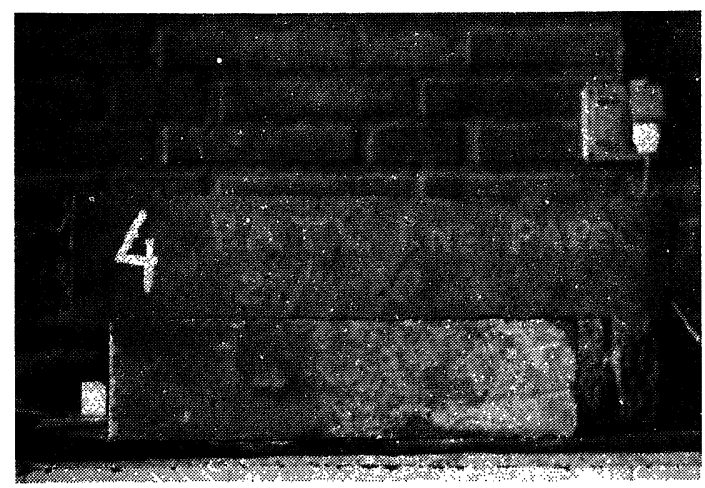

Fotografía 2

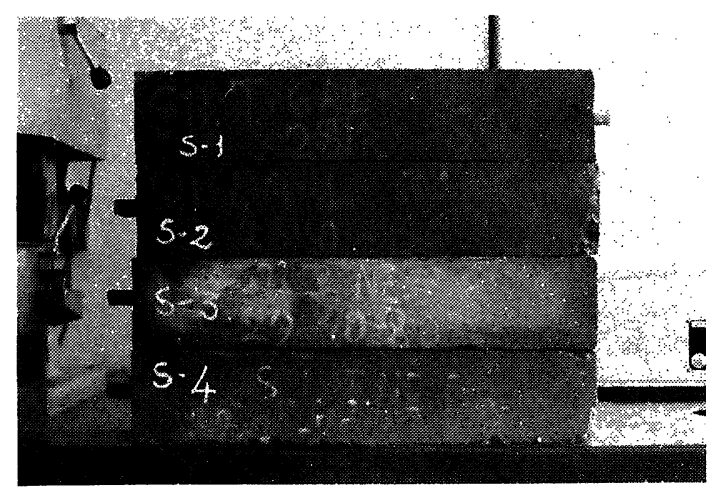

Fotografia 3

\subsection{Preparación para las observaciones}

A la primera fecha de observación (27-XI-81), prácticamente al cabo de 13 meses de la confección de los prismas, se procedió al examen de parte de los mismos.

Para tal fin se cortaron de ellos, mediante una sierra de disco de diamante, trozos de unos $18 \mathrm{~cm}$ de longitud, por el extremo ciego de cada probeta. La porción mayor restante se restituyó a la cámara húmeda para su con servación hasta exámenes posteriores.

La sección resultante del corte en el trozo separado se impregnó con disolución alcohólica de fenolftaleína, con el fin de detectar, por el procedimiento usual, la profundidad de neutralización - bien por carbonatación superficial y poco profunda, o bien por neutralización (pérdida de alcalinidad en toda la masa y espesor de la probeta) debida a posibles interacciones en el seno de la pasta cementante, o bien por ambas cosas a la vez-.

A continuación se separó mediante escoplo la mitad de cada prisma, con objeto de retirar el tubo del hormigón y examinar, tanto la superficie del primero como la huella o hueco dejado por él en el hormigón.

De todas estas etapas se tomaron fotografías en color, cuya observación ahorra el dar descripciones prolijas de las mismas, en cada caso.

\subsection{Observaciones}

De momento, el primer examen al cabo de 13 meses de conservación de los prismas se ha referido sólo a uno de cada dos conservados en las condiciones más desfavorables, es decir, en cámara húmeda, y a los fabricados con los cementos que en una u otra proporción contienen escorias: PA-350 y S-II-350. Los hechos con cemento P-450 y los conservados en ambiente de laboratorio se reservaron para observaciones a más largo plazo.

Las condiciones desfavorables señaladas - $u$ otras bastante próximas o semejantes a ellasno son $\mathrm{ni}$ mucho menos descartables en la realidad, al menos en zonas y épocas determinadas, por lo cual las observaciones hechas en tales condiciones pueden tener un cierto valor práctico.

\section{RESULTADOS}

De acuerdo con las observaciones señaladas en 4.9 y con la preparación para las mismas 
según 4.8, los resultados de dichas observaciones al cabo de 13 meses en los tubos y en las probetas de hormigón objeto de las mismas, han sido los siguientes:

\section{Con cemento PA-350}

Prisma 1 (fotografías 4 y 5):

a) La sección de hormigón es en su totalidad alcalina a la fenolftaleína.

b) El trozo de tubo negro desnudo aparece limpio, prácticamente sin adherencias y no presenta signos de corrosión alguna (la zona roscada ya estaba oxidada inicialmente).

c) La huella del tubo en el hormigón tampoco presenta restos de corrosión del mismo.

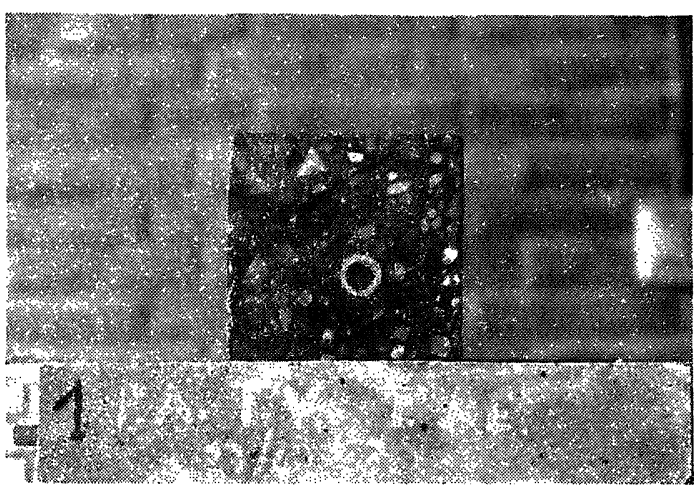

Fotografía 4

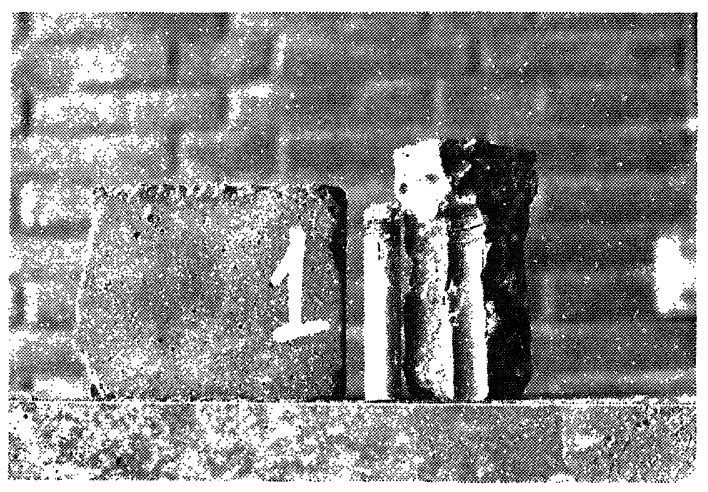

Fotografía 5

Prisma 2 (fotografías 6 y 7):

a) La sección de hormigón es en su totalidad alcalina a la fenolftaleína.

b) El trozo de tubo galvanizado desnudo aparece con algunas adherencias más que en el caso del prisma 1 , pero no presenta ningún signo de corrosión, salvo la de la zona roscada, que ya existía inicialmente.

c) La huella del tubo en el hormigón se muestra sin productos de corrosión del mismo, aunque algo más rugosa que en el caso del prisma 1.

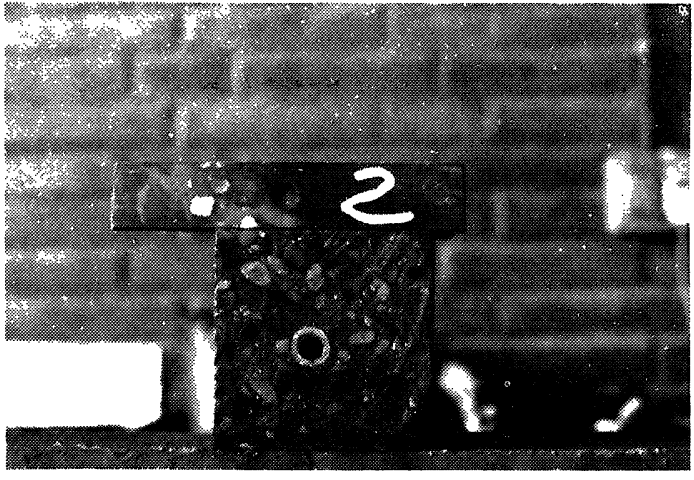

Fotografía 6

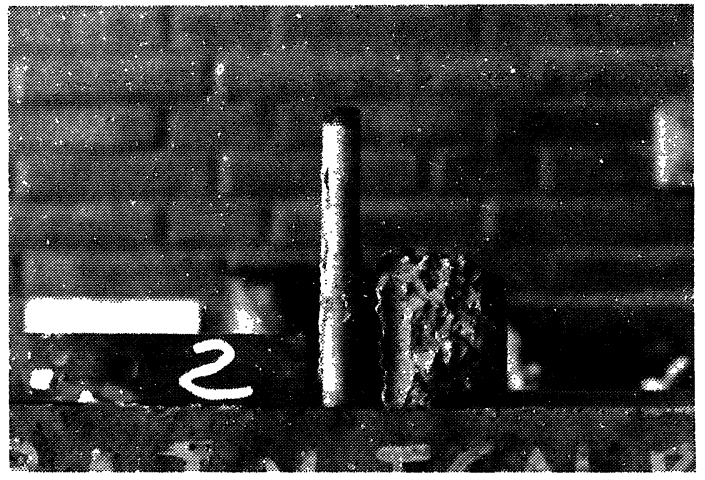

Fotografía 7

Prisma 3 (fotografías 8 y 9):

a) La sección de hormigón es en su totalidad alcalina a la fenolftaleína.

b) El trozo de tubo galvanizado y envuelto en papel de periódico al insertarlo en el hormigón fresco muestra, una vez retirado el papel, haber pérdido en algunas zonas 
el recubrimiento de zinc. En otras lo conserva aparentemente como al principio, y en otras lo presenta alterado y con aspecto distinto. No se observan trazas de herrumbre.

c) La huella del tubo en el hormigón presenta, por zonas, adherencias del papel y de productos de alteración del recubrimiento de zinc que penetran ligeramente en la masa del hormigón. Tampoco aparecen trazas de herrumbre.

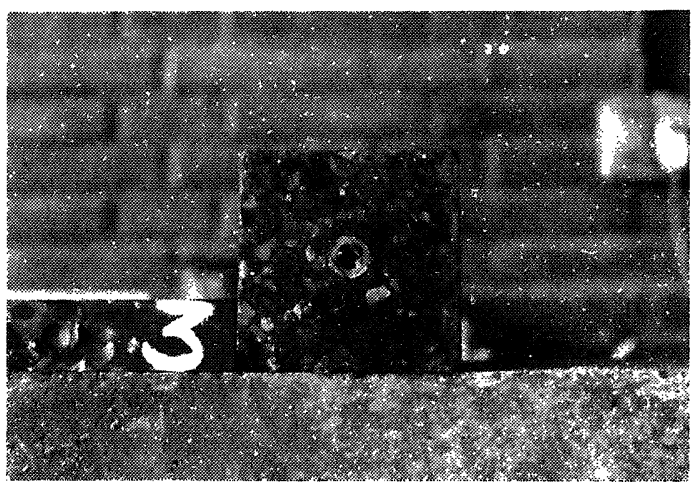

Fotografía 8

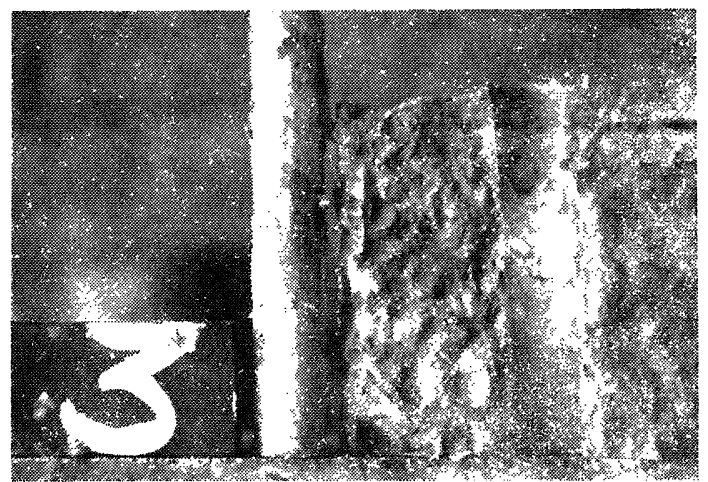

Fotografía 9

Prisma 4 (fotografías 10 y 11):

a) La sección de hormigón es en su totalidad alcalina a la fenolftaleína.

b) El trozo de tubo negro y envuelto inicialmente en papel de periódico, una vez retirado éste, aparece en gran parte limpio y sin signos de corrosión en la zona embebida en el hormigón. En otras zonas presenta adherencias de papel, con algún signo de corrosión.

c) La huella del tubo en el hormigón presenta bastantes adherencias del papel, algunas de ellas con algo de herrumbre.

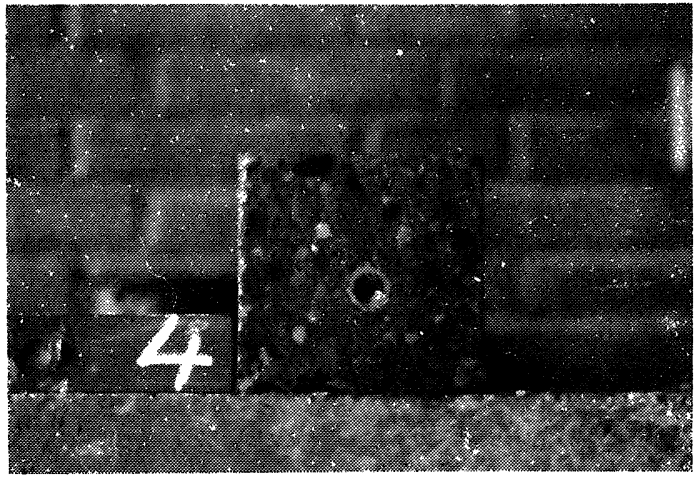

Fotografa 10

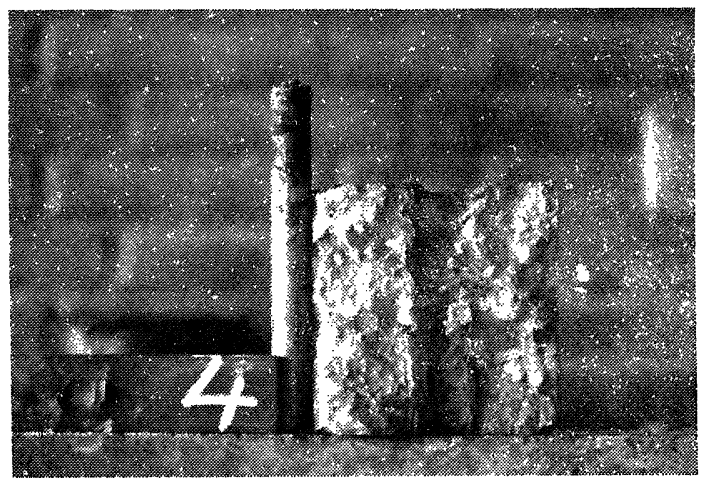

Fotografía 11

\section{Con cemento S-II-350}

Prisma $S 1$ (fotografías 12 y 13):

a) La sección de hormigón es en su totalidad alcalina a la fenolftaleína.

b) El trozo de tubo galvanizado desnudo no presenta señal alguna de corrosión, ni muestra adherencias.

c) La huella del tubo en el hormigón se presenta limpia y con una rugosidad análoga a la del prisma 1. 


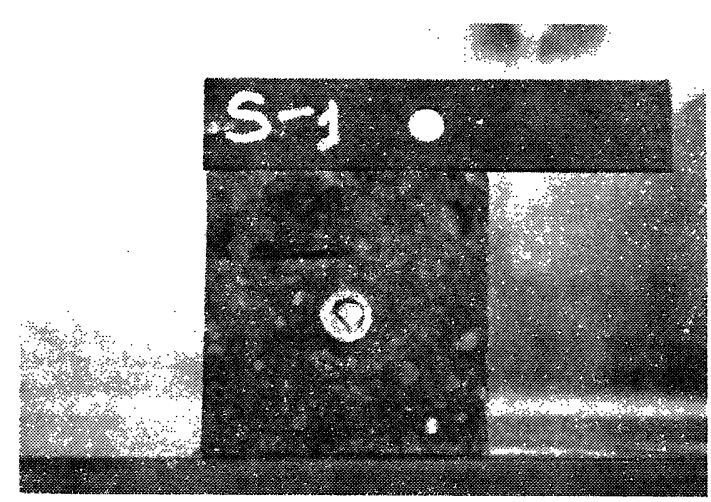

Fotografía 12

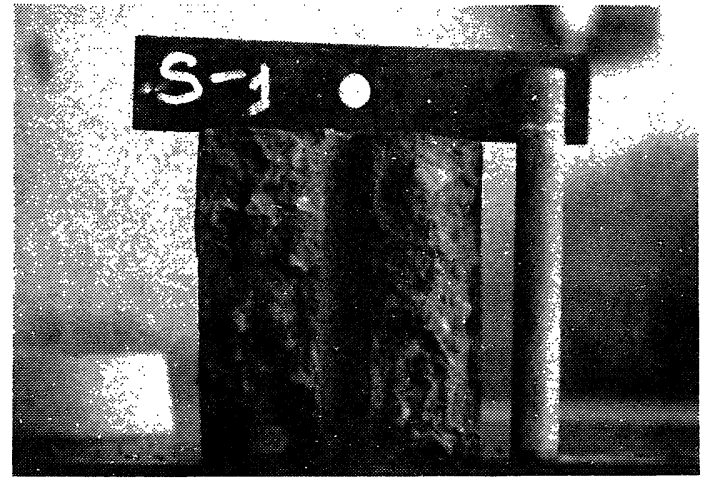

Fotografía 13

Prisma S 2 (fotografías 14 y 15):

a) La sección de hormigón es en su totalidad alcalina a la fenolftaleína.

b) El trozo de tubo galvanizado envuelto en papel de periódico presenta, por zonas, síntomas de alteración y pérdida del galvanizado, aunque no trazas de herrumbre.

c) La huella, del tubo en el hormigón muestra fuertes adherencias de papel en casi toda su totalidad, con mayor deterioro de éste en unas zonas que en otras, aunque no aparecen manchas de herrumbre.

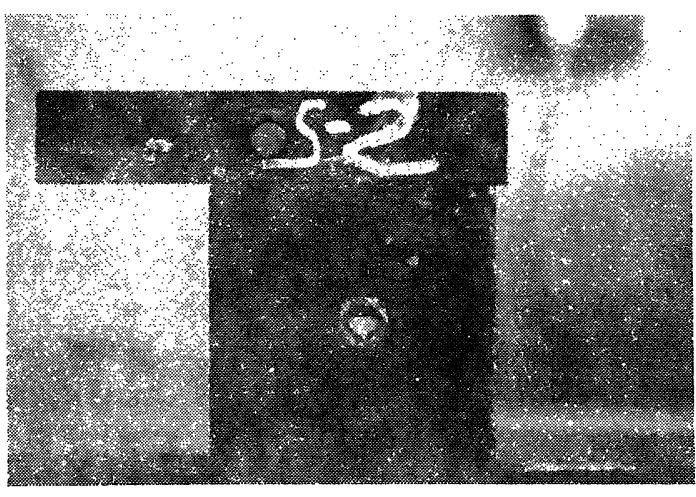

Fotografia 14

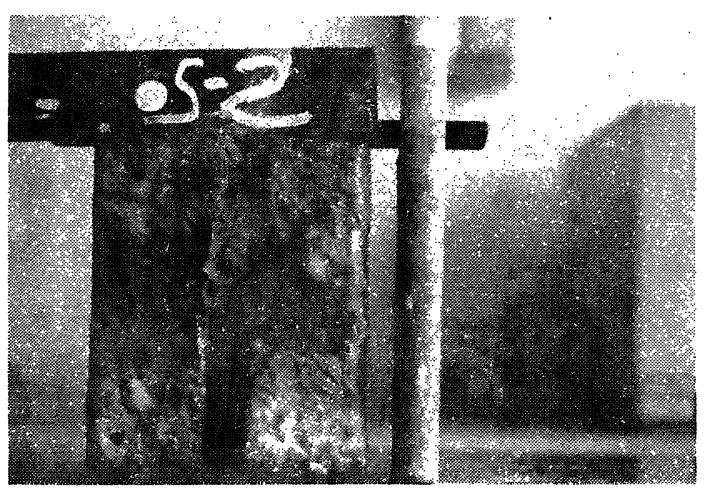

Fotografía 15

Prisma $S 3$ (fotografías 16 y 17)

a) La seción de hormigón es en su totalidad alcalina a la fenolftaleína.

b) El trozo de tubo negro desnudo aparece limpio, apenas con alguna adherencia, y sin signos de herrumbre de corrosión.

c) La huella del tubo en el hormigón se muestra lisa y sin manchas de productos de corrosión.

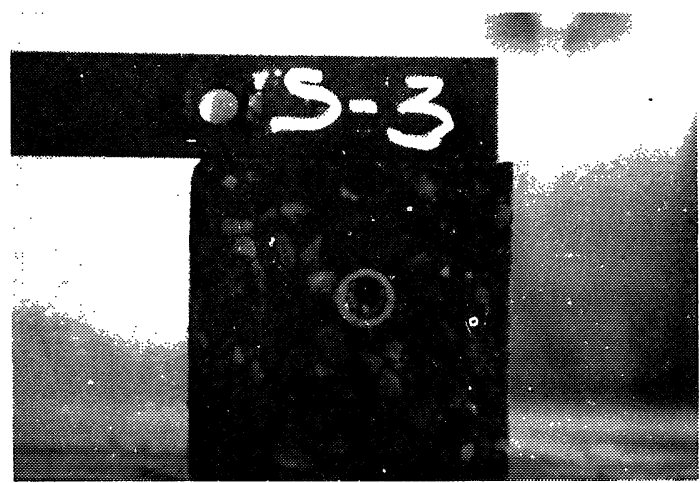

Fotografía 16

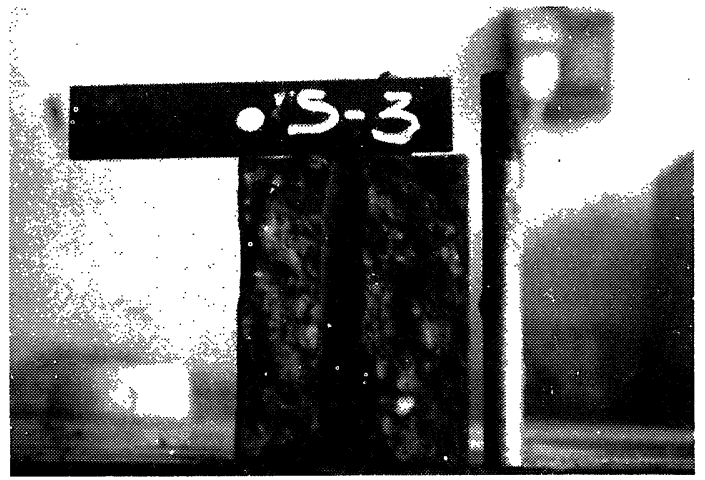

Fotografía 17 


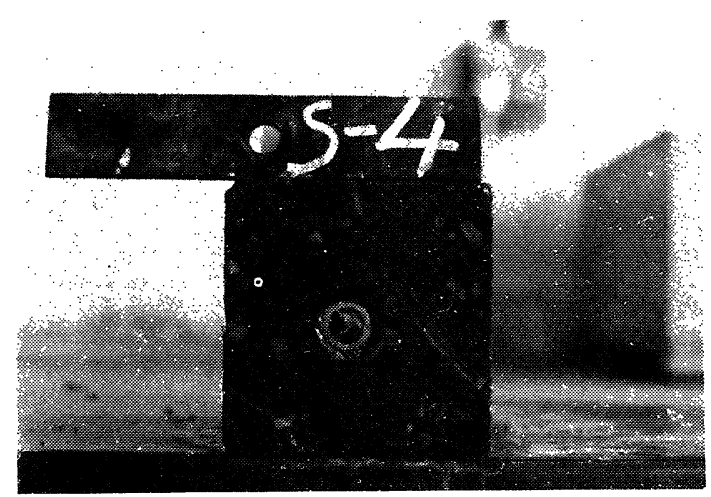

Fotografía 18

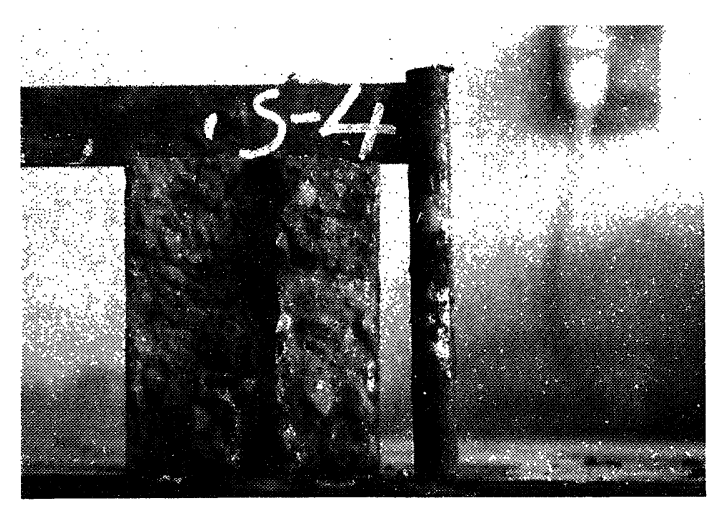

Fotografía 19

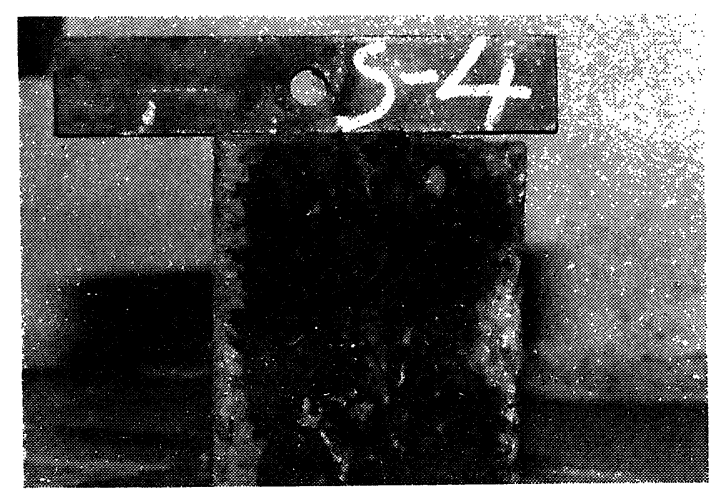

Fotografía 20
Prisma S 4 (fotografías 18, 19 y 20):

a) La sección de hormigón (en este caso tanto frontal como lateral) presenta una zona exterior -estrecha pero de amplitud variable-, neutra o ácida a la fenolftaleína (probablemente debido en parte a carbonatación). El resto mayor de la zona interna de la sección es, en su totalidad, alcalino a la fenolftaleína.

b) El tubo negro envuelto en papel de periódico presenta una acusada corrosión por zonas, con aparición de distintos tonos de herrumbre, desde los pardos claros hasta los oscuros, casi negros, prácticamente a todo lo largo de la longitud del tubo. En otras zonas se observan adherencias.

c) La huella del tubo en el hormigón muestra el papel adherido e impregnado de herumbre en algunas zonas.

\section{INTERPRETACION $Y$ VALORACION DE LOS RESULTADOS}

$\mathrm{Al}$ enjuiciar los resultados observados en los prismas 1 a 4 de la serie correspondiente al hormigón de cemento PA-350, a efectos interpretativos, conviene comparar los de los prismas 1 y 4 (tubo negro, desnudo y envuelto, respectivamente) por una parte, y por otra los de los prismas 2 y 3 (tubo galvanizado, desnudo y envuelto, en cada caso).

En cuanto al tubo negro, su envoltura en papel de periódico no parece ser en absoluto beneficiosa, puesto que ya al cabo de un año parece manifestarse como potencialmente perjudicial al surgir trazas de herrumbre, cosa que no sucede en el caso del tubo desnudo.

En lo que respecta al tubo galvanizado, su envoltura en papel de periódico tampoco parece ser beneficiosa, pues al cabo del mismo período de tiempo, si bien no se aprecian trazas de herrumbre, sí aparece eliminado o alterado por zonas el recubrimiento de zinc, lo que no ocurre con el tubo desnudo.

Parece por ello que la tubería de hierro como tal permanece más protegida en el caso del tubo galvanizado que en el caso del tubo negro durante el período de tiempo de un año, pero nada permite asegurar que al cabo de más tiempo las circunstancias no cambien, dado que el sistema hierro-zinc-hormigón (sin contar con los productos intermedios de la alteración del zinc) es más complejo en sí y en sus posibles evoluciones que el sistema hierro-hormigón, y no es posible prever cómo va a evolucionar. Las observaciones programadas a más largo plazo podrán tal vez ayudar a dilucidarlo. 
Del mismo modo, al enjuiciar los resultados correspondientes a los prismas S 1 a S 4 de la serie del hormigón de cemento siderúrgico S-II-350, conviene comparar, por un lado, los de los prismas S 3 y. S 4 (tubo negro, desnudo y envuelto, respectivamente, como en el caso anterior) y, por otro lado, los de los prismas S 1 y S 2 (tubo galvanizado, desnudo y envuelto, en cada caso, como anteriormente).

Así, pues, en cuanto al tubo negro en este caso, su envoltura previa en papel de periódico resulta aún más perjudicial que en el caso del hormigón de cemento PA-350, puesto que la herrumbre formada por corrosión es mucho más ostensible al cabo del mismo tiempo.

$Y$ en lo que se refiere al tubo galvanizado sucede cualitativamente lo mismo, aunque cuantitativamente tal vez de una forma algo más atenuada. Es decir, las características diferenciales con el tubo negro son las mismas que en el caso del hormigón de cemento PA-350, a saber: la envoltura en papel de periódico parece perjudicar de momento más al tubo negro, aunque su influencia a través de la alteración que produce en el recubrimiento de zinc queda pendiente de observación a plazos más largos.

Por otra parte, la comparación de los resultados de los prismas 1 y 4 , y: 2 y 3 (de la serie de hormigón de cemento PA-350), con los prismas S 3 y is 4, y S 1 y S 2 (de la serie de hormigón de cemento S-II-350), respectivamente, con objeto de hacer destacar la influencia de los cementos, pone de relieve que esta influencia es prácticamente inapreciable al cabo del tiempo de observación de un año, en el caso del tubo negro desnudo; mientras que en el caso del tubo negro envuelto en papel de periódico la influencia del cemento S-II-350 es apreciablemente negativa.

En lo que concierne al tubo galvanizado, la influencia del cemento es prácticamente inapreciable, al cabo de un año, tanto en el caso del tubo desnudo como en el del tubo envuelto en papel de periódico.

Por lo que respecta a la acción y a los efectos del "empapelado" de las tuberías, cabe hacer las siguientes consideraciones:

En primer término, el papel constituye un elemento extraño introducido entre la tubería y el hormigón, el cual evita el contacto entre ambos, afectando a la adherencia natural que en el caso normal se produciría.

En segundo término, el papel es un elemento más o menos absorbente del "agua" del hormigón, que la toma del mismo por imbibición, y que, a través de su masa, como si se tratara de un filtro o de una esponja, la pone en contacto con la superficie del tubo negro o del galvanizado.

Addemás, el "agua" del hormigón es una disolución salina -iónica - con un pH determinado, generalmente del orden de 12,6, tal que en condiciones ordinarias resulta protectora $\multimap$ pasivante y mantenedora del estado pasivo— para el hierro y el acero.

Y en tercer lugar esta disolución salina puede actuar sobre el papel de periódico — sobre su celulosa, sus aprestos, su tinta, etc.-, modificándose por ello sus características, y entre ellas su propio $\mathrm{pH}$. Y esto sin perjuicio de que, como en el caso estudiado, todas las secciones del hormigón aparezcan alcalinas a la fenolftaleína.

En efecto, en el caso presente, y por sugerencia de uno de los autores, se determinó el pH del papel de periódico igual al empleado en la envoltura de los tubos, habiéndose observado que el agua de empapamiento del mismo daba inmediatamente reacción neutra. Pero al cabo de 24 horas el $\mathrm{pH}$ de dicho agua era ya de 6 .

Cabe pensar al respecto en un posible fenómeno de carbonatación, pero también en un 
cambio del $\mathrm{pH}$ provocado por la solubilización de ciertas sustancias contenidas en el papel, sin descartar la posibilidad de ambas cosas simultáneamente. De cualquier manera, la evolución hidrolítica natural de la celulosa es hacia compuestos de carácter más ácido.

En otro orden de cosas, la interposición del papel entre el tubo y el hormigón puede retardar - al menos modificar- la difusión iónica en general, y en particular la de los iones alcalinos, $\mathrm{Ca}^{2+}$ y $\mathrm{OH}^{-}$, lo cual a su vez puede influir en la naturaleza, equilibrio y estabilidad o evolución de la interfase metal/pasta cementante, modificándolos de algún modo, en relación con el caso normal.

Por todo ello, nada tiene de particular que las cosas puedan suceder de distinta manera en el caso de los tubos desnudos que en el de los envueltos en papel, y peor, desde el punto de vista de su corrosión. En tal sentido no hay que perder de vista el hecho de que, a igualdad de todo lo demás, la forma de llevar a cabo la envoltura de los tubos con papel de periódico puede tener, por lo indicado en lo que precede, su propia influencia.

\section{\%. CONCLUSIONES PROVISIONALES}

Como conclusiones provisionales deducidas de una primera observación de las probetas - hormigón y tubos - al cabo de 13 meses de conservación en cámara húmeda, se pueden emitir las seis siguientes:

7.1. El cemento PA-350 conteniendo escoria siderúrgica (la específica del cemento estudiado) como adición activa, así como el cemento siderúrgico S-II-350 (conteniendo la suya específica), ambos utilizados en el presente estudio, no parecen haber ejercido efectos corrosivos, ni sobre la tubería de acero negro desnudo, ni sobre la galvanizada desnurda, al cabo de un año.

7.2. Con el cemento PA-350 utilizado parece haberse producido una corrosión muy ligera al cabo de un año, sobre el tubo negro envuelto en papel de periódico, mientras que la corrosión producida en las mismas condiciones por el cemento S-II-350 parece ser más notoria.

7.3. Ni el cemento PA-350 ni el S-II-350 utilizados hacen aparecer herrumbre al cabo de un año sobre el tubo galvanizado envuelto en papel de periódico, aunque tanto en uno como en otro caso aparecen alteraciones y pérdidas parciales, prácticamente de la misma magnitud, en el recubrimiento de zinc del tubo galvanizado.

7.4. Todo lo anterior indica que, a efectos de corrosión del tubo negro, o de pérdida parcial o alteración del recubrimiento de zinc del tubo galvanizado, parece tener mucho mayor influencia - negativa- el hecho de envolver previamente los tubos en papel de periódico, que la naturaleza - contenido de escorias- de los cementos.

7.5. Se considera preciso alargar más el período de curado para la observación de las probetas, con objeto de comprobar si se mantiene la ausencia de corrosión en los tubos negros y galvanizados desnudos, y si progresan, tanto las corrosiones incipientes observadas sobre el tubo negro envuelto en papel, como las pérdidas y alteraciones del recubrimiento de zinc en el tubo galvanizado, asimismo envuelto en papel.

7.6. Se juzga igualmente necesario comparar a más largo plazo éstos y posteriores resultados con los de los tubos embebidos en hormigón de cemento P-450, así como con los de los tubos de probetas conservadas en ambiente de laboratorio. 


\section{RECOMENDACIONES PROVISTONALES}

A la vista de los resultados, de su interpretación y valoración, y de las conclusiones emanadas de todo ello, parece inmediato deducir que la práctica de envolver los tubos, tanto negros como galvanizados, en papel de periódico antes de su recubrimiento por morteros u hormigones de los cementos hasta ahora considerados aquí (PA-350 con escoria - la suya específica- y S-II-350 siderúrgicos con su escoria también específica), es perjudicial para la conservación de las tuberías.

Es opinión extendida que la práctica de envolver con papel las tuberías de agua en la edificación, constituye una solución económica de compromiso entre, por una parte, la conveniencia de dejar alrededor de las mismas un pequeño espacio que permita absorber fácilmente los cambios limitados de volumen que pudieran experimentar por efecto de las variaciones de temperatura en el caso de las de calefacción y agua caliente, por razón de sus condiciones de servicio; y también porque de esta forma se evita que la pared externa de las tuberías esté en contacto con un medio demasiado heterogéneo, como en la práctica resultan ser los materiales de relleno en los que se alojan las tuberías horizontales (materiales de composición y de características físicas -porosidad, compacidad-diferentes). Y, por otra parte, el inconveniente que supone a veces la existencia de esos pequeños espacios de aire alrededor de las tuberías, los cuales pueden dar lugar en ocasiones a la formación de pilas de aireación diferencial. Claro está que una solución, sin duda de mayor efectividad para la conservación de las tuberías, es la de colocarlas dentro de una funda o vaina antes de su puesta en obra y en contacto con los materiales de construcción, prescripción ésta que es la que adopta la normalización francesa -DTU $60.1-(2)$.

Pero, en todo caso, el papel que se utilice para envolver las tuberías no debe ejercer ninguna acción química sobre la pasta de cemento ni sobre ellas mismas, condición que no cumple el papel de periódico -o algún papel de periódico-, tal como queda de manifiesto en este trabajo.

Por otra parte, la práctica demuestra que ciertos tipos especiales de papel ejercen, sobre todo en condiciones de sequedad, un cierto efecto protector sobre las tuberías, cuando en obra son envueltas con ellos; éste es el caso del papel de las bolsas usadas de cemento portland, en razón de su impermeabilidad y de la alcalinidad que en húmedo puede conferirles el polvo de cemento retenido en sus poros (2); y también el de ciertos papeles comercializados, impregnados con, o como soporte de, determinados preparados protectores anticorrosivos idóneos que, aplicados oportuna y adecuadamente, pueden dar resultados positivos, incluso evitando muchas veces el recubrimiento de los tubos con mortero u hormigón, y protegiéndolos "al aire", como por ejemplo en empotramientos verticales de conducciones en edificación.

Pero para ello es imprescindible tanto la oportunidad y la adecuación del recubrimiento, como la buena práctica y ejecución del mismo o de la pintura -adherencia, poder de cubrimiento, etc.-, pues, de lo contrario, podrían resultar asimismo ineficaces o perjudiciales.

Conviene tener presente que los hormigones confeccionados para esta investigación son de laboratorio, muy cuidados y de buena calidad, como ponen de manifiesto las fotografías. En todo caso son de calidad bastante mejor que la de la gran mayoría - por no decir la totalidad- de los hormigones o morteros de relleno que, en general, cubren - con frecuencia deficientemente- a las tuberías en edificación. Por lo tanto, los resultados encontrados en este estudio, tanto hasta la fecha como en el futuro, pueden no ser extrapolables a la realidad práctica. 
Visto de otra manera, lo anterior quiere decir que si los resultados encontrados hasta ahora en este trabajo se juzgan como buenos - en los casos de tubos desnudos-, su repetición en la práctica exige, con toda seguridad, los mismos cuidados en cuanto a los materiales y a la ejecución. Y quiere decir también que, en caso de resultados insatisfactorios, en la realidad probablemente lo serian mucho más.

Por lo tanto, se recomienda que los morteros y hormigones de relleno y recubrimiento de tuberías estén bien dosificados, sean homogéneos, rodeen por completo a los tubos en todas sus partes sin dejar huecos ni discontinuidades, y sean compactos e impermeables en la mayor medida posible. En suma, se recomienda extremar las precauciones, por otra parte usual y normalmente observadas - pero en todo caso obligadas-, respecto de la idoneidad de los materiales empleados y, por encima de todo, cuidar escrupulosamente la ejecución, en lo que concierne a la colocación, recubrimiento y empotramiento de los tubos. Se debe entender que, en este aspecto como en tantos otros de la tecnología de la construcción, y más que en muchos, es bastante más barato prevenir que curar.

\title{
R E F E R E N C I A S
}

(1) RC-75: "Pliego General de Condiciones para la Recepción de Cementos". Ministerio de Obras Públicas y Urbanismo, Madrid, 1975.

(2) C. DEr Ormo: Comunicación privada, 1982.

\section{próxima publicación del IETcc}

\section{BASES PARA EL DISEÑO SOLAR PASIVO}

Equipo de Investigación de Ahorro de Energía en el Edificio (IETcc)

\begin{abstract}
Las dificultades de suministro y el alto coste de los productos energéticos convencionales han despertado la atención de los usuarios, técnicos e industriales de la edificación hacia los procedimientos y sistemas en que se basa el aprovechamiento de otras fuentes alternativas de energia, principalmente la solar. Esto ha generado un rápido desarrollo industrial y comercial que, en opinión de los autores de este libro, arrastran los siguientes defectos: un mimético tecnologismo respecto de los sistemas convencionales que violenta las peculiaridades de la energia solar (baja densidad y variabilidad en el tiempo), y una escasa selectividad en la aplicación de los sistemas y procedimientos pasivos dando origen a un ecumenismo arquitectónico solar, al margen de las condiciones climáticas y funcionales especificas de cada caso y lugar.

En este libro, utilizando criterios y metodologia pedagógicos, se dan los fundamentos e instrumentos teórico-prácticos necesarios para el planteamiento de todo proyecto arquitectónico solar pasivo, de acuerdo con los principios éticos y económicos de conservación y ahorro de energia. Es decir: respeto de los presupuestos bioclimáticos, búsqueda de la máxima captación y acumulación de la radiación solar, y esmero en el aislamiento térmico de los cerramientos.

Este libro contendrá los siguientes capitulos: Situación energética en el sector edificación. El hombre y. el clima. El edificio Cálculo del soleamiento. Captación solar. Acumulación de calor. Aislamiento térmico. Análisis económico. Fichas de recomenda-
\end{abstract} ciones prácticas. Anexos. Bibliografia. 